\title{
Ultra Short Pico Second Chirped Pulse Generation using SESAM based Mode- Locked Fiber Laser
}

KEYWORDS : Mode-locking, chirp, timebandwidth product, Semiconductor Saturable Absorber Mirror (SESAM).

\section{ABSTRACT}

Ultra short pulse fiber lasers are widely used in many photonic systems for industrial, biomedical and scientific applications. Popularity of these lasers rapidly developed due to increment in demand of shorter pulses for various applications like communication, ophthalmology, micromachining, medical imaging and precision metrology. Pulsed fiber laser can produce ultra short pulses in order of pico or femto second. Mode locking technique is widely used in rare earth doped fiber lasers to produce such ultra short pulses of light. In this paper, pulsed operation of fiber laser was studied experimentally at 1 micron region. Experiment on pulsed fiber laser has been done using ytterbium $(Y b)$ doped active fiber. Using the principle of passive mode locking, a 2.3 pico-second pulse was produced at 1064nm wavelength. A semiconductor saturable absorber mirror was used to mode lock the laser. The spectral domain data showed that the pulse was not Fourier transform limited which means the pulse was chirped. Chirped pulse amplification systems exploit this pulse characteristic for power scaling of ultra-short pico-second to femto-second pulses.

\section{Introduction}

Various advantages of fiber laser make them popular over semiconductor lasers in different application fields. The waveguide structure provides light guidance over very long distance and long interaction path of light with the active medium. Alignment free laser system design is possible using narrowband reflectors (known as Fiber Bragg Grating or FBG) directly inside the fiber and splitting or combining light beams in a single fiber. High beam quality of laser output is achieved by single mode light propagation. Extraction of heat is possible due to the large surface to active volume ratio of fibers which increase the power handling capability of fiber laser. Efficient doping of rare earth elements in fiber core enables the active fibers to be operated at very high average power.

Active fibers with small core diameter and small acceptance angle can't accept the pump light efficiently. The successful implementation of clad pumping technique overcomes this limitation of fiber laser. In clad pumping technique, two cladding is present in fiber. The outer cladding is made by low refractive index materials (polymer glass or air) than that of inner cladding. Large diameter of inner cladding with relatively large index difference between inner and outer cladding makes the inner cladding ideal for pump guidance. After launching the pump beam into the inner cladding, it is confined within it by outer cladding. The pump is progressively absorbed into the core as it is propagated along the fiber [1].

Ytterbium $(\mathrm{Yb})$ is belongs to the group of rare earth metals. In laser technology, $\mathrm{Yb}$ is used in the form of trivalent ion $\mathrm{Yb}^{3+}$. Core of the optical fibers are doped by $\mathrm{Yb}$ ions to make the fiber active and these active fibers are used as active gain media of the fiber laser system. Absorption peaks for $\mathrm{Yb}$ present at $915 \mathrm{~nm}$ and $975 \mathrm{~nm}$ whereas emission peaks are at $975 \mathrm{~nm}$ and $1030 \mathrm{~nm}$. Due to the emission band around 1030 to $1100 \mathrm{~nm}$, this range is treated as the gain bandwidth for $\mathrm{Yb}$ doped media. The gain bandwidth of $\mathrm{Yb}$ is the wavelength range over which laser gain is obtained. The metastable state lifetime for $\mathrm{Yb}$ is $850 \mu \mathrm{s}$ [2]. $\mathrm{Yb}$ doped fibers can be operated at $1064 \mathrm{~nm}$ and pumped at $976 \mathrm{~nm}$.

Ultra short pulse generation with high peak power using pulsed operation of fiber laser becomes popular due to the increment in demand of shorter pulses in various fields. Chronologically, more than $100 \mathrm{~W}$ average power was achieved from ultra short pulse fiber lasers in 2005 and in 2009 it reaches almost 1kW [3].

\section{Time-Bandwidth Product (TBP) and Chirped Pulse Ampli-} fication (CPA)

Any pulse has a certain Full Width Half Maximum (FWHM) value in temporal domain. The Fourier transformed spectra of that pulse also has a certain FWHM value in the spectral domain.
The product of these two parameters is called the Time Bandwidth Product (TBP) of the pulse. For a particular pulse shape, pulses have unique TBP values. For Gaussian pulse, TBP is 0.4413 and for second hyperbolic pulse, TBP is 0.3148 [4]. If the TBP value of a pulse is maintained then the pulse is called transform limited pulse. Transform limited pulses don't change their pulse shape. Chirp is the instantaneous frequency change in the pulse envelope. Often the output pulses coming from a mode locked fiber laser are chirped. Chirped pulses don't maintain their TBP value and hence they are no more transform limited. Pulse shape of such pulses is changed due to the effect of chirp.

Largest possible pulse energy can be achieved using Chirped Pulse Amplification (CPA) technique. Output pulses coming from the mode locked fiber lasers are often chirped. CPA systems exploit these pulses characteristics for power scaling of ultra-short pico- second to femto-second pulses. CPA can generate sub picoseconds pulses with pulse energies of around 1 micro joule. Such low pulse energies are sufficient for many applications [5].

\section{SESAM based Mode-Locking for Ultra Short Pulse Genera-} tion

Saturable absorber is a medium whose absorption decreases with increment of light intensity. Semiconductor materials which can absorb over a broad range of wavelength are used as saturable absorbers. SESAM is a saturable absorber that operates in reflection. In SESAM, a highly reflective surface is covered by the saturable absorber. At low intensities, saturable absorber absorbs most of the incident light and the reflectivity of the mirror is small. If the incident intensities increase, the saturable absorber absorbs less and mirror reflectivity increases. When SESAM used as a mirror in a laser cavity, high intensity portions have a higher feedback compared to lower intensity portions. This feature enables high intensity pulse formation process. In case of fiber lasers, Semiconductor based saturable absorbers are made of InGaAsP or GaAs. They can be grown on top of reflecting multilayer structures and hence referred as semiconductor saturable absorber mirror (SESAM). Use of SES$\mathrm{AM}$ in the fiber laser cavity makes the laser system more compact and stable. Response times of such SESAMs are in the sub Pico second range.

In a laser cavity, a laser oscillates with many longitudinal modes simultaneously. There is no fixed phase relationship exists between the different longitudinal modes. The output is the sum of the intensities of each individual mode and hence has random fluctuations in amplitude. If we locked the phases of each oscillating modes to bring them all in phase at any time and maintain this phase relationship then due to redistribution of electric field, high intensity pulse form in the cavity. To lock the phases 
of the modes, we can choose the boundaries of the cavity where the different oscillating modes are in phase. SESAM is placed at one of the boundary of the cavity. When a pulse with random fluctuations in intensity comes to the SESAM, SESAM absorbs the lower intensity peaks of the pulse and gradually saturates. Thus only the highest intensity peak of that pulse reflects back to propagate through cavity and achieves gain from the active medium. The relaxation time of the SESAM is the time needed by a SESAM to return in its re-absorbing state. If the round trip time taken by the pulse is greater than the relaxation time of the SESAM then after one round trip in the cavity, the pulse comes again to the SESAM and similar things happen because meanwhile the SESAM is ready for re-absorption. Thus the output of the laser is a repetitive series of pulses of light with a repetition rate $\mathrm{c} / 2 \mathrm{~nL}$ where $\mathrm{L}$ is the cavity length and $\mathrm{n}$ varies as $1,2,3 \ldots$ Such pulse train called mode locked pulse train. This technique is called SESAM based mode locking. The relaxation time of the SESAM is an important parameter in SESAM based mode locking technique because if the relaxation time of SESAM is shorter, shorter pulses are obtained.

Mode locking technique is widely used in rare earth doped fiber lasers to produce ultra short pulses of light. Broader gain bandwidth of the laser, shorter pulse duration is achieved. For a $40 \mathrm{~nm}$ gain bandwidth at $1550 \mathrm{~nm}, 5 \mathrm{THz}$ corresponding frequency bandwidth is obtained. If modes over this bandwidth are mode locked then achievable pulse duration becomes $200 \mathrm{fs}$ [6]. Thus fiber lasers are very useful for generation of ultra short pulse using mode lock technique.

\section{Mode-Locked Fiber Laser using Yb doped Active Fiber}

The experimental set up for the mode lock fiber laser system was consists of a semiconductor laser diode for pumping, a wavelength division multiplexer (WDM), a semiconductor saturable absorber mirror (SESAM), a FBG, an isolator and a splitter. Power meter was connected for power measurement and optical spectrum analyzer (OSA) was used for spectrum analysis.

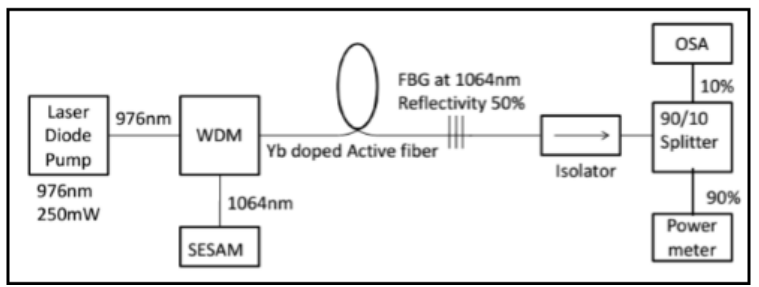

Figure 1: Experimental set up for mode lock fiber laser system (SESAM based)

JDSU manufactured semiconductor laser diode with maximum power $250 \mathrm{~mW}$ was used as a pump source at $976 \mathrm{~nm}$. WDM was used to discriminate the propagation of signals with different wavelengths. The $976 \mathrm{~nm}$ port was used for pump signal propagation and the $1064 \mathrm{~nm}$ ports were connected to the active fiber and SESAM. 50\% reflective Teraxian manufactured FBG with center wavelength $1064 \mathrm{~nm}$ and $1 \mathrm{~nm}$ bandwidth and the Batop manufactured SESAM together made the resonator cavity of the mode lock fiber laser system. Due to the partial transitive nature, laser output extracted from the FBG. In house made $\mathrm{Yb}$ doped single clad fiber was used as active medium of the laser system. Length of active fiber was taken as $0.8 \mathrm{~m}$. Thor labs manufactured isolator was used to protect the other devices from the back reflection. OZ optics manufactured 90/10 splitter splits its own input into two parts. $90 \%$ was going to the power meter and $10 \%$ was going to the OSA. Splitting was necessary to protect the OSA from high power. Power meter measured the average power of the output laser pulse and OSA was used to study the spectrum of the pulse in terms of power vs. wavelength. The spectral FWHM of the output pulse also measured from the
OSA.

Oscilloscopes can be used to measure the pulse width of the laser output in temporal domain. But if the pulse has very narrow pulse width i.e. in range of pico or femto second, oscilloscopes are not efficiently able for pulse width measurement. In such cases, Autocorrelators are used to measure the pulse width of such ultra short pulses. From the autocorrelation trace, we get the exact pulse width of the ultra short pulse in order of femto second for a fixed pump power.

\section{Experimental Results}

Intensity of laser output with respect to wavelength was measured in OSA for $100 \mathrm{~mW}, 150 \mathrm{~mW}, 200 \mathrm{~mW}$ pump powers. Continuous wave (CW) component was obtained at $1064 \mathrm{~nm}$ for high pump power. The FWHM (in wavelength scale) of the laser output also measured in OSA for different pump powers. Autocorrelation trace was taken at various pump power. Pulse width obtained around $2.3 \mathrm{ps}$ at $150 \mathrm{~mW}$ pump power.

\section{Conclusions}

From the experimental results of the mode lock laser, we can conclude that due to the increment in pump power, the intensity of laser output also increases. Under higher pump power, a CW peak was observed over the pulse spectra. Intensity profile gets broaden for higher values of pump power. This is happens because in presence of high pump power the peak power of the pulse increases and due to this the nonlinear effect Self Phase Modulation (SPM) broaden the pulse [7].

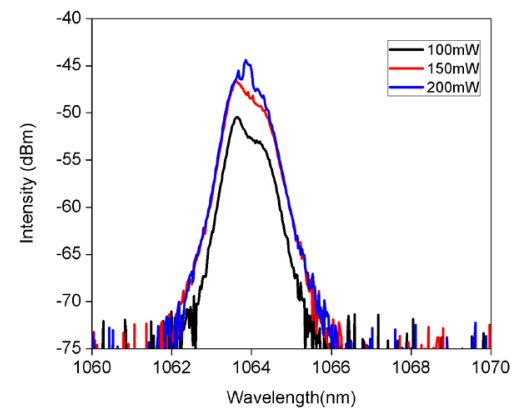

Figure 2: Intensity of laser output for various pump power

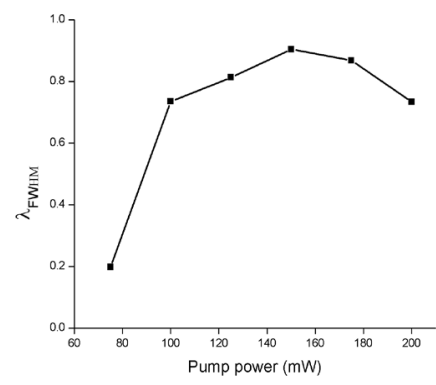

Figure 3: $\lambda_{\text {FWнм }}$ of laser output for various pump power

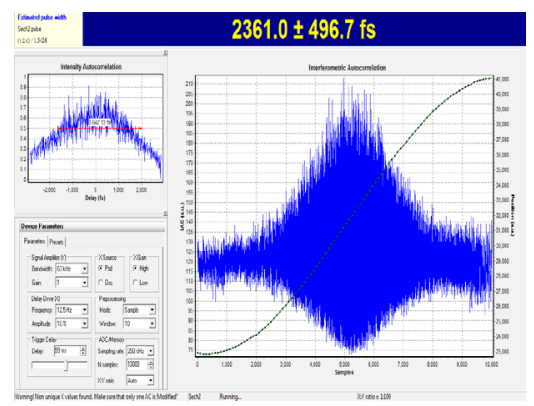

Figure 4: Autocorrelation trace of laser output 
In SPM effect, new frequency components are generated continuously as the pulse propagates through the fiber. These SPM generated frequency components broaden the spectrum over its initial width. The chirp induced by SPM increases in magnitude with propagated distance. The shape of the spectrum also deviate from the Gaussian profile which implies that the pulse was chirped. The initial increment in $\lambda_{F W H M}$ was due to the SPM effect. But when CW peak was obtained, the measured $\lambda_{F W H M}$ values were dropped. From the autocorrelation trace, we obtained the pulse width of the mode lock generated pulse. As $\lambda_{F W H M}$ is known along with pulse width, we can calculate the TBP of the pulse and this information helps us to understand whether the mode lock generated pulse is chirped or not.

Now at $150 \mathrm{~mW}$ pump power,

$\lambda_{F W H M}=0.9039 \mathrm{~nm}$ and pulse width $=2.3 \mathrm{ps}$.

So $\Delta T=2.3 \times 10^{-12} \mathrm{~s}$ and $d \lambda=0.9039 \times 10^{-9} \mathrm{~m}$

As $f \lambda=c$

Or, $d f=-\frac{c}{\lambda^{2}} d \lambda$

Or, $d f=-\frac{3 \times 10^{8} \times 0.9039 \times 10^{-9}}{\left(1064 \times 10^{-9}\right)^{2}}$

Or, $d f=-2.3953 \times 10^{11} \mathrm{~Hz}$

The minus sign signifies that a positive difference in wavelength will cause a negative difference in frequency but their magnitude is proportional.

Now $\Delta T=2.3 \times 10^{-12} \mathrm{~s}$

So TBP is $\quad \Delta T d f=0.5509$

For Gaussian pulse, TBP is 0.4413 and for second hyperbolic pulse, TBP is 0.3148 [4].

As 0.5509 is greater than the TBP of a transform limited pulse, the laser output surely a chirped pulse.

\section{Acknowledgement}

I would like to express my heartiest gratitude to Dr. Mrinmay Pal, Senior Scientist, Fiber Optics and Photonics Division (FOPD), CSIR-Central Glass and Ceramic Research Institute (CGCRI), for his valuable guidance and co-operation to complete this work. I am also thankful to the Director of the CSIR-CGCRI for giving me the opportunity to use the CGCRI laboratory and scientific software for my work.

\section{REFERENCE}

